\title{
A Better Performance to INPE Satellite Control Software
}

\author{
Andreia Carniello*, Adriana Carniello ${ }^{\dagger}$, Mauricio G. V. Ferreira ${ }^{\ddagger}$, José Demisio S. da Silva ${ }^{\S}$ \\ National Institute for Space Research, São José dos Campos, SP, 12227-010, Brazil
}

\begin{abstract}
A very important issue in distributed systems is the management of load among system nodes. Load balancing enables a better use of the system resource capabilities and a better performance by allocating nodes that are more suitable for the execution of some tasks. This work proposes a multi-agent load balancing architecture for distributed object applications called MALBA which uses artificial neural networks and a set of policies to support the process of migrating and replicating the application objects. MALBA architecture outlines a decentralized and run-time balancing process that balances the load among nodes which execute a distributed object application.
\end{abstract}

\section{Introduction}

$\mathrm{T}$ HE availability of low-price microprocessors and the progress of the communication technology have increased the interest in distributed systems. The main advantages of these systems are high performance, availability of resources, and extensibility at a low cost. Taking into account these advantages, many organizations have adopted distributed systems and the use of distributed object applications.

Distributed objects are independent programs that might be located at any node of a network and might be accessed by remote clients via method invocation. Clients do not need to know where objects are located, i.e., if objects are located at the client's node or not ${ }^{1}$. Shortly, in a Distributed Object application the objects reside on different machines and communicate remotely and transparently to one another to execute their functionalities. The objects offer services and communicate by requesting services to one another. The requester is called the client and the provider is the server. We propose a load balancing architecture to balance applications built under this paradigm.

In a distributed system, service requests arrive randomly at the nodes. This might generate a non-balanced state of the system that may lead to the existence of overloaded nodes and idle ones. This situation may harm services response time by the application objects, as well as, the usage of system resources. Dynamic load balancing involves the reallocation of the application objects to nodes after their initial assignments. This is done by migrating objects from the overloaded nodes to other lightly loaded nodes to improve the overall system performance ${ }^{2}$.

The existence of a load balancing service avoids some nodes to get overloaded while others become idle. In this paper, the main concern is how to distribute the application objects among nodes for decreasing the application execution time and for optimizing the use of system resources.

Thus we propose a load balancing architecture called MALBA - Multi-Agent Load Balancing Architecture for Distributed-Object Applications, which is formed by different agents working together to provide a balanced solution for the system. Agents feel the environment and act on it by migrating objects from overloaded nodes to idle nodes and also by replicating highly requested objects at less busy nodes in order to get a more balanced distribution of objects and consequently balancing the system load.

Task migration could be either preemptive or nonpreemptive ${ }^{8}$. Preemptive task migration allows tasks in execution to be stopped, transferred, and then resumed at another node. Non-preemptive migration allows only tasks that have not yet started execution to be transferred. Preemptive transfer incurs more overhead than the

\footnotetext{
* Doctorate student, Applied Computing Postgraduate Program (CAP), Av. dos Astronautas 1758 Jd. Granja, ancarnie@lac.inpe.br.

$\dagger$ Doctorate student, Applied Computing Postgraduate Program (CAP), Av. dos Astronautas 1758 Jd. Granja, adcarnie@lac.inpe.br.

\$ Doctor Researcher, Satellite Control and Tracking Center (CRC), Av. dos Astronautas 1758 Jd. Granja, mauricio@ccs.inpe.br.

$\S$ Doctor Researcher, Applied Mathematics and Computing Associated Laboratory (LAC), Av. dos Astronautas 1758 Jd. Granja, demisio@lac.inpe.br.
} 
nonpreemptive counterpart. Hence, it should be used only if the performance gains outweigh the transfer costs. Our approach employs non-preemptive migration.

The difference between replicating and migrating an object is that in replication the object is kept at its local node and a copy of it is instantiated at a remote node whereas in migration the object is removed from its local node and it is instantiated at a remote node.

The main focus of this work is the policies we propose for the load balancing process, which are performed by the agents of MALBA architecture. An artificial neural network based agent classifies the system nodes in load levels. An agent makes decision as to balance the system by statistically analyzing the load levels of nodes. The selection of objects to migrate and the nodes to receive these objects is supported by migration policies which are performed by an agent. For the replication of objects some policies are used to guide the selection of objects to be replicated and the nodes that will receive the replica. An agent performs the replication of the objects and an agent removes the replicas that are not in use anymore.

MALBA architecture follows a decentralized approach that means every node might take balancing decisions, thus implying in a non-centralized balancing control. As each node has autonomy to do the balancing, if a failure occurs at one node that does not put in risk the load balancing service.

This paper is organized as following: Section II introduces general features of MALBA architecture. Section III describes the Time-based Load Balancing Service. Subsection A describes the load checking service, and in Subsection B and C we present the object migration and replication policies, respectively. In Subsection D we describe the load balancing execution service. Section IV introduces the Request-based Load Balancing Service and in Section V some results are discussed. In Section VI we present some related works and we draw some conclusions in Section VII.

\section{MALBA Architecture}

MALBA architecture applies two approaches to balance the system nodes: the Request-based Load Balancing Service and the Time-based Load Balancing Service, both illustrated in Figure 1. The first is a load balancing service which is activated when a request arrives at the system. When a client object requests a service to a server object, the load balancing is activated to select the most appropriate node to execute the server. This selection is done according to some policies that will be explained next in this paper. So, this approach worries at keeping the system balanced at each server execution.

Whereas the Request-based approach activates the load balancing at every new request, the Time-based approach activates the load balancing at random and it evaluates whether the system is balanced or not, and in case it is not balanced some policies are applied to balance the system load. These policies will be explained next. So, this approach aims at adjusting the system load from time to time.

Next we present both approaches in details, the Time-based and the Request-based one.

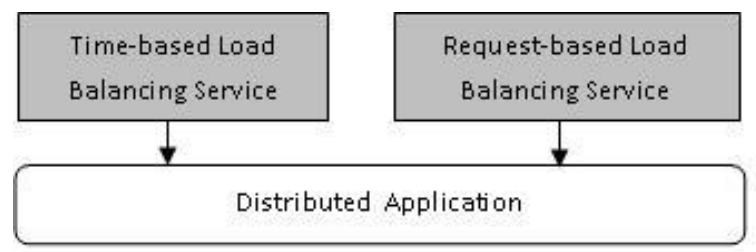

Figure 1. MALBA load balancing architecture.

\section{Time-based Load Balancing Service}

Figure 2 illustrates the Time-based Service which comprises four other services: the system load checking service, the load balancing execution service and the services related to the policies for migrating and replicating the application distributed objects. 


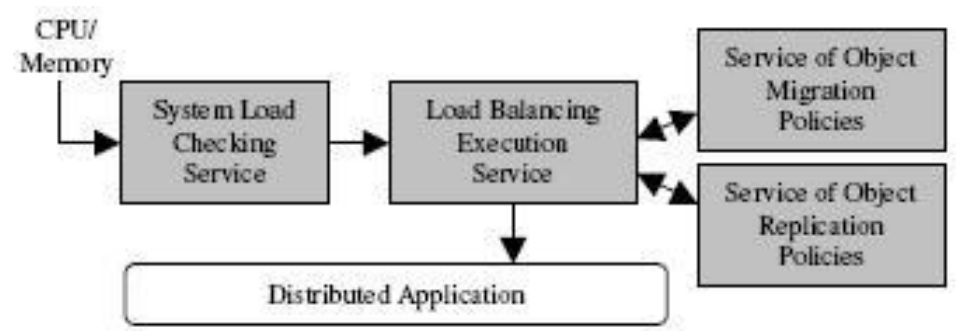

Figure 2. Time-based load balancing service.

In the following subsections we detail these services.

\section{A. Load Checking Service}

The load balancing is activated at random and every system node has autonomy to do so. The first step of the balancing process is to perform the Load Checking Service which is responsible for classifying the nodes in load levels and also for checking if there is a need to do the balancing. Two agents are in charge of performing this service: the Load Classifier Neural Agent and the System Load Checker Agent.

Load Classifier Neural Agent - the Neural Agent is responsible for stating the load level of the system nodes and it is present in all nodes. This agent collects two load indexes from a node: CPU usage and memory usage, and classifies this node in one of the following levels - (level 1): strong idle; (level 2): idle; (level 3): normal load; (level 4): overload; and (level 5): strong overload. For instance, the set $\{3,1,5,4,2\}$ represents the possible load levels of a system with five nodes.

The Load Classifier Neural Agent uses an MLP neural network (Multiple Layer Perceptron) for classifying the load level of the system nodes. Our neural network was trained in a supervisioned way by the backpropagation algorithm ${ }^{3}$. Further details on our implementation is described in Section 3.

System Load Checker Agent - it has as its input the load level information generated by the Neural Agent. The Load Checker Agent checks whether the system is load balanced or not. Just in case the system is not balanced, it activates the balancing process.

First, the Load Checker Agent calculates the standard deviation $(\sigma)$ considering the load level of all the system nodes, as follows:

$$
\sigma=\sqrt{\frac{\sum_{i=1}^{n}\left(n c_{i}-\overline{n c}\right)^{2}}{n-1}}
$$

where:

- $n c_{i}$ represents the load level of node $i$ (provided by the Load Classifier Neural Agent);

- $\overline{n c}$ represents the load level average of the $n$ nodes of the system;

- $n$ represents the amount of nodes in the system.

Once the Load Checker Agent has calculated the standard deviation $(\sigma)$, it applies the function bal $(\sigma)$ which defines whether the balancing process will be activated or not according to an activation threshold. Function $\operatorname{bal}(\sigma)$ is as follows:

$$
\operatorname{bal}(\sigma)= \begin{cases}1 & \text { if } \quad \sigma>\frac{\sigma_{\max }}{4} \\ 0 & \text { otherwise }\end{cases}
$$


where:

- $n$ represents the amount of nodes in the system;

- $\sigma_{\max }$ represents the maximum value of the standard deviation for $n$ nodes.

If the result of the standard deviation $(\sigma)$ is greater than $1 / 4$ of the standard deviation maximum value $\sigma_{\max }$, then the load balancing process will be activated, otherwise it will not.

The standard deviation maximum value $\sigma_{\max }$ is calculated considering all the nodes of the system ( $n$ nodes) and the threshold value to activate the load balancing process represents $1 / 4$ of this value, i.e., $\sigma_{\max } / 4$.

The activation threshold was defined empirically taking into account the fact that as closer to zero the result of the standard deviation is, more balanced is the load of the overall system, and as the standard deviation gets far from zero, more non-balanced the overall system is and, consequently, there might be a need to activate the load balancing process.

\section{B. Service of Object Migration Policies}

Once the Load Checking Service has identified the need to activate the load balancing process, the distributed application, running at that moment, will have its object reallocated to better balance the overall system load.

Objects located at overloaded nodes should be migrated to idle nodes. The Service of Object Migration Policies is responsible for performing the choice of objects to migrate and the choice of nodes to receive these objects. These two selection processes are performed by the Migration Object Selector Agent and by the Migration Node Selector Agent, respectively.

Migration Object Selector Agent - The first action of the Object Selector Agent is to select the load dispatcher node $\left(N_{\text {dispatcher }}\right)$, i.e., the most overloaded node in the system:

$$
N_{\text {dispatcher }}=\arg \max _{i}\left\{n c_{i}\right\} \quad i=1,2,3, \ldots, n
$$

where:

- $n c_{i}$ represents the load level of node $i$ (provided by the Load Classifier Neural Agent);

- $n$ represents the amount of nodes in the system.

Once a load dispatcher node $\left(\mathrm{N}_{\text {dispatcher }}\right)$ has been selected, the Object Selector Agent selects among its objects the ones which are not in use by the running application, i.e., the objects with no active connection, that is, objects with null connection, which are candidate objects to migrate because an object can only be migrated if it is not executing any of its services.

In the present discussion, $C$ will denote the set of candidate objects to migrate and $C_{i}$ will denote an object of this set.The set of candidate objects to migrate $(C)$ is determined by the Object Selector Agent as follows:

$$
C=\arg \underset{i}{\operatorname{Con}}\left(\left\{O N_{i}=0\right\}\right) \quad i=1,2, \ldots, n
$$

where:

- $n$ represents the amount of objects in the $\mathrm{N}_{\text {dispatcher }}$ node;

- $O N_{i}$ represents the object $i$ of $N_{\text {dispatcher }}$ node;

- $\operatorname{Con}\left(\left\{O N_{i}\right\}\right)$ represents the connection of object $O N_{i}$.

Once the Object Selector Agent has identified the candidate migrating objects $(C)$, the next step is to quantify, for each candidate object, the following features:

- the size of the candidate object (in bytes);

- the amount of relations between the candidate object and the $N_{\text {dispatcher }}$ node objects;

- the amount of relations between the candidate object and the objects remote to $N_{\text {dispatcher }}$ node.

A relation between two objects occurs when one of the objects calls for a service provided by the other. If an object (obj1) relates with $n$ other objects, the amount of objl relations is $n$. 
Among the candidate objects, the Object Selector Agent identifies an object that satisfies the criteria listed below.

(i) the object which is the biggest in size (number of bytes);

(ii) the object with the least amount of relations with the $N_{\text {dispatcher }}$ node objects;

(iii) the object which has the greatest amount of relations with the objects located remotely from the $N_{\text {dispatcher }}$ node.

The Object Selector Agent selects the migrating object as the one that satisfies the greater amount of the criteria listed before.

(i)

The Object Selector Agent identifies, from candidate objects of set $C$, the object (denominated $i^{o b}$ ) which has the biggest size, as follows:

$$
i^{o b}=\arg \max _{i}\left(\operatorname{Tam}\left\{C_{i}\right\}\right) \quad i=1,2, \ldots, n
$$

where:

$n$ represents the amount of objects of set $C$;

$\operatorname{Tam}\left\{C_{i}\right\}$ represents the size of object $C_{i}$.

(ii)

Among the candidate objects in set $C$, the Selector Agent identifies the object (denominated $j^{o b}$ ) which has the least amount of relations with the $N_{\text {dispatcher }}$ node objects, as follows:

$$
j^{o b}=\arg \min _{i}\left(R I\left\{C_{i}\right\}\right) \quad i=1,2, \ldots, n
$$

where:

- $n$ represents the amount of objects of set $C$;

- $R I\left\{C_{i}\right\}$ represents the amount of object $C_{i}$ relations with objects of set $O N$.

(iii)

The Object Selector Agent identifies, from candidate objects of set $C$, the object (denominated $k^{o b}$ ) which has the greatest amount of relations with the objects located remotely from $N_{\text {dispatcher }}$ node, as follows:

$$
k^{o b}=\arg \max _{i}\left(R E\left\{C_{i}\right\}\right) \quad i=1,2, \ldots, n
$$

where:

- $n$ represents the amount of objects of set $C$;

- $R E\left\{C_{i}\right\}$ represents the amount of object $C_{i}$ relations with objects remote to $N_{\text {dispatcher }}$ node.

Once $i^{o b}, j^{o b}$, and $k^{o b}$ have been defined, the Object Selector Agent creates a histogram for each object in the $N_{\text {dispatcher }}$ node representing the object frequency in set $\vartheta=\left\{i^{o b}, j^{o b}, k^{o b}\right\}$.

The set $O N$ represents all the objects in $N_{\text {dispatcher }}$ node and considering $r \in O N$, the histogram $H_{r}$ is defined as follows:

$$
\begin{aligned}
& H_{r}=\sum_{q=1}^{3} 1_{r}(\vartheta(q)), \\
& 1_{r}= \begin{cases}1 & \text { if } \vartheta(q)=r \\
0 & \text { otherwise }\end{cases}
\end{aligned}
$$


The Object Selector Agent finally defines the object to migrate $\left(O_{\text {migrate }}\right)$ as follows:

$$
O_{\text {migrate }}=\arg \max _{t}\left(\left\{H_{t}\right\}\right) \quad t=1,2, \ldots, n
$$

where:

- $n$ represents the amount of objects in $N_{\text {dispatcher }}$ node.

Migration Node Selector Agent - Once the object to migrate $\left(O_{\text {migrate }}\right)$ has been selected, the Node Selector Agent chooses the most suitable node to receive this object.

The first step of the Node Selector Agent is to select the node which has the least load in the system, based on the load level of nodes. In case there are nodes with the same least load level then a decision criterion is used to select the node to receive the object $O_{\text {migrate }}$ as the one with the greastest amount of relations with the object $O_{\text {migrate }}$.

\section{Service of Object Replication Policies}

According to Ferreira ${ }^{4}$, object replication benefits the load balancing of the overall system because it can relieve the load of a node by replicating its highly requested object at another node, implying that new service requests will be answered by the replicated object.

The Service of Object Replication Policies is responsible for leading the selection of the object to replicate and the selection of the replica receiver node. These selections are performed by the Replication Object Selector Agent and by the Replication Node Selector Agent, respectively. The former selects from the candidate objects (which are the objects in the most overloaded node that have active connections, i.e., the objects in use at the moment) the one which has the greatest amount of active connections to be the object to replicate. The node to receive this replica is selected by the latter agent as the one with the least load level in the system or in case there are nodes with the same least load level the one which has the greatest amount of active connections with the object to replicate.

\section{Load Balancing Execution Service}

The Load Balancing Execution Service is responsible for reconfiguring the distribution of objects of the application in order to get a more load-balanced system. This service is formed by the Object Migrator Agent, the Object Replicator Agent, and the Replica Remover Agent.

Object Migrator Agent - This agent is responsible for migrating objects from overloaded nodes to idle nodes.

This agent communicates with the agents of the Migration Policies Service in order to be aware of the object to migrate and the load receiver node. Based on these perceptions, the Object Migrator Agent performs the object migration.

Object Replicator Agent - This agent is responsible for replicating objects from overloaded nodes to idle nodes.

This agent communicates with the agents of the Replication Policies Service in order to be aware of the object to replicate and the replica receiver node. Based on these perceptions, the Object Replicator Agent performs the object replication. Once replication occurs, all the future requests to the replicated object will be answered by its replica, because the replica has higher priority than the original object.

Replica Remover Agent - This agent is responsible for removing object replicas, in overloaded nodes, which are no longer in use (connection $=0$ ).

\section{Request-based Load Balancing Service}

The main goal of the Request-based Load Balancing Service is decomposed according to the goal decomposition diagram in Figure 3. The main goal "Keep the system load balanced when executing new requests" is decomposed into sub-goals "Execute new requests in the server node when its load level is not classifications 4 and 5" or "Execute new requests in the client node when its load level is not classifications 4 and 5" or "Execute new requests in a selected system node". The main goal is satisfied when one of the three sub-goals is satisfied. Thus, the goal "Keep the system load balanced when executing new requests" is satisfied when: (1) the new request is executed in the server node when its load level is not overloaded; or when (2) the new request is executed in the client node 
when its load level is not overloaded; or when (3) the new request is executed in any other node of the system that is not overloaded.

Goal 1 on its turn is satisfied when the arrival of the requests in the server node is monitored and when the load of the server node is classified. Goal $\mathbf{2}$ is satisfied when the load of the client node is classified and when the server object is migrated to the client node. Goal $\mathbf{3}$ is satisfied when a node that is not overloaded is selected to receive the server object.

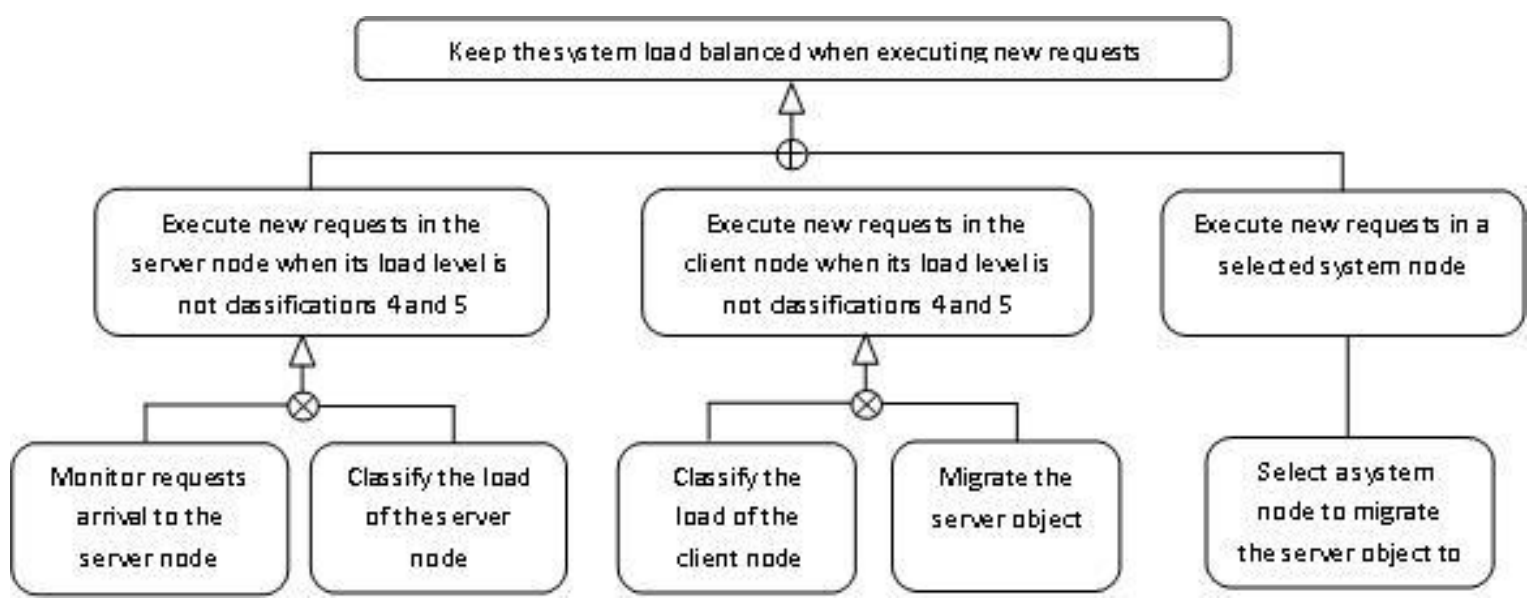

Figure 3. Goal decomposition diagram for the request-based load balancing service.

\section{Tool and Results}

A prototype tool is under development to realize the MALBA architecture project. We have currently implemented MALBA Load Checking Service which is executed by the Load Classifier Neural Agent and the System Load Checker Agent.

In order to implement the Neural Agent, we firstly practiced some knowledge engineering to associate CPU and memory usages to the five load levels defined in Subsection A. Table 1 presents these associations.

Table 1. CPU and memory usages associated to our five load levels.

\begin{tabular}{|c|c|c|c|c|c|}
\cline { 2 - 6 } \multicolumn{1}{c|}{} & \multicolumn{5}{c|}{ MEMORY } \\
\hline CPU & $0-0.19$ & $0.2-0.39$ & $0.4-0.59$ & $0.6-0.79$ & $0.8-1.0$ \\
\hline $0-0.19$ & Level 1 & Level 2 & Level 3 & Level 4 & Level 5 \\
\hline $0.2-0.39$ & Level 2 & Level 2 & Level 3 & Level 4 & Level 5 \\
\hline $0.4-0.59$ & Level 3 & Level 3 & Level 3 & Level 4 & Level 5 \\
\hline $0.6-0.79$ & Level 4 & Level 4 & Level 4 & Level 4 & Level 5 \\
\hline $0.8-1.0$ & Level 5 & Level 5 & Level 5 & Level 5 & Level 5 \\
\hline
\end{tabular}

The Load Classifier Neural Agent uses an MLP neural network (Multiple Layer Perceptron) which has been trained in a supervised way by the error backpropagation algorithm ${ }^{3}$. The network architecture is formed of 02 entries, 37 neurons in the hidden layer and 03 neurons in the output layer, having as their activation function the hyperbolic tangent and the sigmoid logistic, respectively.

The training set had 10201 inputs and in 30000 epochs the network achieved an error of 0.000008 .

The Neural Agent outputs, i.e., the load level of system nodes, are the entries to the Load Checker Agent which uses these values to statistically verify whether it is necessary to balance the system load or not. For further details on the Load Checker Agent behaviour see Subsection A.

After training the Neural Agent and coding the Load Checker Agent behavior, tests were conducted to validate MALBA architecture tool. Table 2 shows the specification of two test cases. The execution of these test cases produced the expected results presented in Table 2. 
Table 2. Test cases executed for testing MALBA architecture Load Checking Service.

\begin{tabular}{|c|c|c|c|c|c|}
\hline Test & \multicolumn{3}{|c|}{ Test Data } & \multicolumn{2}{|c|}{ Expected Results } \\
\hline \multirow{4}{*}{01} & Node & $\begin{array}{l}\text { CPU } \\
\text { Usage }\end{array}$ & $\begin{array}{c}\text { Memory } \\
\text { Usage }\end{array}$ & $\begin{array}{c}\text { Node Load } \\
\text { Level }\end{array}$ & $\begin{array}{l}\text { Balancing } \\
\text { Decision }\end{array}$ \\
\hline & 1 & 0.92 & 0.63 & 5 & \multirow{3}{*}{$\begin{array}{c}\text { Activate load } \\
\text { balancing }\end{array}$} \\
\hline & 2 & 0.31 & 0.27 & 2 & \\
\hline & 3 & 0.78 & 0.64 & 4 & \\
\hline \multirow{3}{*}{02} & 1 & 0.57 & 0.46 & 3 & \multirow{3}{*}{$\begin{array}{l}\text { Do not activate } \\
\text { load balancing }\end{array}$} \\
\hline & 2 & 0.71 & 0.58 & 4 & \\
\hline & 3 & 0.53 & 0.37 & 3 & \\
\hline
\end{tabular}

In Test Case 01 execution, the Neural Agent output was the following load levels: 5, 2, 4. The standard deviation of the system node load levels was 1.247219128924647 and the maximum standard deviation for this threenode system was 1.8856180831641267 . Thus, the balancing decision was to activate load balancing. In Test Case 02, the Neural Agent output was 3, 4, 3, the standard deviation was 0.4714045207910317 and the maximum standard deviation was 1.8856180831641267 . Thus, the decision was not to activate load balancing.

A relevant issue is that MALBA architecture considers that the system nodes are homogeneous, i.e. all the nodes are equivalent in their hardware and software architectures. This allows the application objects to be located at any system node. Besides that, nodes are considered to be equivalent in their processing and storage capabilities.

\section{Related Works}

Load balancing is a classical problem and there is an extensive literature on it.

Suri, T'oth and Zhou in Ref. 5 consider load balancing as a game. There is a set of clients, each of whom must choose a server from a permissible set. Each client has a unit-length job and selfishly wants to minimize its own latency (job completion time). This interaction was modeled by the authors as an atomic congestion game which is part of game theory.

Nogueira, Yamin, Vargas and Geyer in Ref. 6 present a framework developed with the objective of assisting designers of high performance distributed applications to construct, simulate, and understand clearer the impact caused by the balancing decisions in the system general performance.

MALBA migration and replication policies consider communication costs related to local objects and also to remote objects. The idea is to locate objects that communicate to each other, i.e., objects that call other object services, together in the same node in the system to reduce the remote communication costs. In Ref. 7 these policies are not defined and in Ref. 2 they are defined, however the authors consider just the communication costs among local tasks.

In Ref. 8, a taxonomy of approaches to the distributed scheduling problem is presented in an attempt to provide a common terminology and classification mechanism necessary in addressing this problem. MALBA architecture belongs to the dynamic distributed classification described in Ref. 8, which consists of making balancing decisions at run time and the work involved in making these decisions is physically distributed among the nodes. This descentralized approach makes MALBA a safe load balancing service because there is not the existence of a unique failure point in the system whereas non-distributed load balancing approaches ${ }^{9,7,10}$ might put load balancing in risk if the central node fails.

\section{Conclusions}

MALBA architecture has been designed to provide system load equilibrium by a better distribution of the objects in a distributed application. MALBA load balancing service is performed in a decentralized and dynamical way, redistributing the load among system nodes at execution time. This redistribution is based on a set of policies which leads the object migration/replication process. These policies are guidelines to select objects to migrate/replicate and also to select the recipient nodes of these objects.

In MALBA architecture, the load balancing is performed by means of agents that communicate to each other to take balancing decisions. This multi-agent architecture uses artificial neural networks, by means of a neural agent, to classify the load level of system nodes. 
In order to validate MALBA architecture, we intend to apply the proposed ideas to a distributed object application such as the Prototype of INPE (National Institute for Space Research) Satellite Control System. Optimizing the use of INPE computational resources is a mean for the Control Satellite System to be improved to support additional new satellites, operating at computational conditions that avoid overloads.

\section{Acknowledgments}

This work was financially supported by Coordination of Postgraduate Personnel Improvement (CAPES) from the Brazilian Government.

\section{References}

${ }^{1}$ Purao, S., Jain, H. K., and Nazareth, D. L., "Effective Distribution of Object-Oriented Applications," Communications of the ACM, 41(8), 1998, pp. 100-108.

${ }^{2}$ El-Abd, A., and El-Bendary, M., "Neural-Based Selection and Location Policies for Dynamic Load Balancing in Distributed Computing Systems," Proceedings of the IASTED International Conference on Modeling and Simulation, 1998.

${ }^{3}$ Haykin, S., Neural Networks - A Comprehensive Foundation, Macmillan, New York, 1994.

${ }^{4}$ Ferreira, M. G. V., "A Dynamic and Flexible Architecture for Distributed Objects applied to Satellite Control Software," Doctorate Thesis in Applied Computing Postgraduate Program, INPE, São José dos Campos, Brazil, 2001.

${ }^{5}$ Suri, S., T'oth, C. D., and Zhou, Y., "Selfish Load Balancing and Atomic Congestion Games", Algorithmica, SpringerVerlag, New York, Vol 47 , No 1, 2007, pp. $79-96$

${ }^{6}$ Nogueira, M. L. B., Yamin, A. C., Vargas, P. K., Geyer, C. F. R., "Load Balancing in Distributed Systems: An Evaluation Environment Proposal", XXVII Latin-American Conference on Informatics (CLEI 2001), Mérida, 2001.

${ }^{7}$ Yang, J., Jizhou, S., and Zunce, W., "Load Balance in a New Group Communication System for the WAN," CCECE CCGEI, IEEE, Montreal, 2003.

${ }^{8}$ Casavant, T. L., Kuhl, J. G., “A Taxonomy of Scheduling in General-Purpose Distributed Computing Systems”, IEEE Transactions on Software Engeneering, Vol 14, No 2, 1988, pp. 141-154.

${ }^{9}$ Schlemer, E., "A Scheduling Solution for DPC++," Master Dissertation in Computer Science, UFRGS, Porto Alegre-RS, Brazil, 2002.

${ }^{10}$ Alvim, R., Grossmann, F., and Dantas, M., "Implementation of a Distributed Architecture with High Availability for Linux,” Electronic Journal of Scientific Initiation, Brazilian Computer Society, Vol. 2, No. 3, 2002. 